\title{
Interactive comment on "An efficient two-layer landslide-tsunami numerical model: effects of momentum transfer validated with physical experiments of waves generated by granular landslides" by Martin Franz et al.
}

Anonymous Referee \#2

Received and published: 20 February 2020

\section{Summary:}

The Authors present a numerical study into subaerial granular landslide-tsunamis. The numerical model is a two-layer model based on the shallow-water equations, validated with a range of experiments from Miller et al. (2017). A range of rheological slide models is tested with the Coulumb rheology identified as the best option to model the observed laboratory deposits. The wave amplitudes are investigated at several locations along a flume and compared with the experimental results as well as with predictions based on an empirical equation from the technical literature.

Printer-friendly version

Discussion paper 
The topic fits well within the scope of NHESS. The approach (elastic collision principle) to model the interface between the slide and water layer is interesting and I can imagine that the Authors invested a lot of time to implement and test the model. Unfortunately, I do not think the selected application (landslide-tsunamis) was a good choice as the applied shallow-water equation model excludes key physical principles for tsunami generation and propagation such as frequency dispersion. This is the reason why shallow-water equation models (in the form used by the Authors) were state-ofthe-art 15-10 years ago. The model may be valuable for engineering applications and to speed up the prediction process, however, this is supposed to be a research article. A research article should enhance the physical understanding of a phenomenon. The selected equations by the Authors (the shallow-water equations) are not only outdated for landslide-tsunami modelling, but the Authors also introduce unphysical aspects (e.g. the thin water layer to avoid singularities/numerical issues, the introduction of the fitting parameter in Eq. (31), the unphysical elastic collision principle (L184), the unphysical water surface plot in the last frame of Fig. 4, the back analysis in the Voellmy rheology (258), etc.) and the Authors often fall short in making a precise interpretation of the reason for the mismatch between numerical and experimental results.

In addition, the agreement between numerical and experimental results is rather modest (see e.g. Figs. 9 and 11). Further, I feel that some of the Conclusions contradict the findings in the article (e.g. the Authors praise the good fit of their data in Fig. 11 to the prediction given by Eq. (39) (L417), or they claim that the numerical waves agree well with the experimental one (L410), which is not at all confirmed in Fig. 9, and there is in addition no proper method applied to quantify the numerical-experimental agreement (such as nRMSE)). Strongly related references representing the current state-of-theart achieving a much better agreement are not includes in the Introduction (e.g. Ma et al. (2015)) and also the presentation of the Figures needs to be significantly improved.

The article requires a lot of work for all these issues to be addressed and to communicate the weaknesses clearer. Below are some more Specific comments together with

Printer-friendly version

Discussion paper

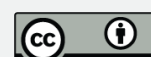


a list with Grammar issues and minor points. I recommend a major revision where all these points are carefully addressed.

Specific comments:

A motivation for the Author to use the simplified model is to save computational resources (L44). Therefore, it would be good to discuss this aspect in the manuscript (e.g. what were the used computer resources, what was the computation time of typical run, what is the cell size, etc.)

L19: A landslide can also be partially submerged.

L38: Smoothed Particle Hydrodynamics is a solver, not a set of governing equations. Modelling the generation of subaerial landslide-generated tsunamis with the shallowwater equations was state-of-the-art 15-10 years ago. I feel the way this is written is slightly misleading. Further, more appropriate governing equations for the problem are the RANS equation with turbulence closure, amongst others (LES, DNS, Euler equations).

L41: It is unclear what the Authors mean by "full 3D", Smoothed Particle Hydrodynamics can also address the problem in 3D? Also, coupled approaches are a common trend nowadays, which are not covered, see e.g. Tan et al. (2018). A numerical landslidetsunami hazard assessment technique applied on hypothetical scenarios at Es Vedrà, offshore Ibiza. Journal of Marine Science and Engineering 6(4):1-22 and other two-layer models such as Ma et al. (2015). A two-layer granular landslide model for tsunami wave generation: Theory and computation. Ocean Modelling 93:40-55. See also the comprehensive review of Yavari-Ramshe and Ataie-Ashtiani (2016). Numerical modeling of subaerial and submarine landslide-generated tsunami wavesâĂTrecent advances and future challenges. Landslides for a more holistic overview of numerical options.

L41: What is a less classical hybrid approach? Please elaborate.

Printer-friendly version

Discussion paper 
L43: Why do the predictive methods do need to be numerical, why not physical model studies other methods?

L45: Again, the application of the shallow-water equations for landslide-tsunami generation, at least for subaerial ones, was state-of-the-art around 15 years ago as they exclude, in the general form, key physical concepts such frequency dispersion. It should also be clearer from the text which shallow-water equations are discussed (nonhydrostatic, non-linear, linear)?

L50: It is unclear what the Authors mean by "drag-like equations", please give more details.

L59: "method is obviously wrong from a physical perspective". This is supposed to be a research article, not an Engineering report, so the introduction of physical flawed concepts is slightly questionable. Please comment.

L86: Again, the Authors need to be more precise which shallow-water equations they apply. Some of them are appropriate, at least for tsunami propagation. None of them is appropriate for subaerial landslide-tsunami generation in my view.

L120: The descriptions in the text does not fit with the term "relative density", which indicates one density relative to the other, rather than one density minus another density. The sentence after ("Since each. ..") does not resolve this confusion well.

L163: I understand that the Authors introduce a small water layer to avoid zeros in the water depth array. In other words, the Authors introduce unphysical boundary conditions to avoid singularities/a numerical issue. This needs a better justification in my view. The fact that this water layer is transferred into a viscous layer does not really resolve this questionable approach. A better option to deal with a numerical issue is on the numerical side, not by compromising the physics.

L184: More unphysical behavior is introduced with the elastic collision principles, research should give new physical insight into phenomena, not to opposite. I invite the

Printer-friendly version

Discussion paper 
Authors to comment.

Eq. (31): A fitting parameter is introduced. How would the solution look like without fitting parameters? This is a weakness that the maximum slide needs to be known a priori to compute the slide behavior and landslide-tsunami afterwards. Can the Authors discuss where they would know this parameter from in a real scenario?

L226: I am not sure if the Reader is interested in a comparison which is difficult to performed. It looks like this came out of a discussion within the Author team. And it would be better suited in the Discussion section. I suggest removing this paragraph.

The water surface elevation in the last screenshot in Fig. 4 looks rather unphysical.

L250: The Authors aim to predict landslide-tsunamis in nature, by fitting their numerical result to the one in the laboratory. How do the Authors deal with the fact that laboratory granular slide deposits do not represent the behavior in nature at all due to scale effects (see e.g. Kesseler et al. (2020). Grain Reynolds number scale effects in dry granular slides. Journal of Geophysical Research-Earth Surface 125(1):1-19.)? Would their model still be able to predict the phenomenon in nature?

L258: In the Voellmy rheology a back analysis is performed to fit the laboratory experiment. How would this be done if applied to real cases?

L271: "same order of magnitude" means up to a factor of 10 difference. Is this really what the Authors try to state? How useful is a numerical simulation modeling the phenomenon with a difference of up to a factor of 10 ? Further, in Fig. 5 the mass between the physical and numerical slides seems to be very different. Is the mass at all conserved in the numerical model?

L323: It is unfortunate that the Authors include 5 and $10 \mathrm{~cm}$ water depth in their analysis, which are cases in the region of significant scale effects (see the technical literature where $h>0.200 \mathrm{~m}$ is specified to avoid significant scale effects for granular slides). The model does not include all physics to represent scale effects (e.g. surface

Printer-friendly version

Discussion paper 
tension). This is a limitation of the study. E.g. how can the Authors be sure that scale effects are not responsible that their simulation fits well with the experimental results at NHESSD $5 \mathrm{~cm}$ water depth?

Fig. 9: The Authors need to apply a parameter to proper judge the agreement between the laboratory and numerical results, e.g. a normalised Root Mean Square Error (nRMSE) relative to the amplitude, or similar.

Interactive

comment

Fig. 9: The weaknesses of the shallow-water equation approach become now obvious in the wave profiles, e.g. frequency dispersion is not modelled resulting in significant deviations between numerical and laboratory results. A detailed discussion about frequency dispersion is required, see e.g. Ruffini et al. (2019) Numerical modelling of landslide-tsunami propagation in a wide range of idealised water body geometries. Coastal Engineering 153:103518 or similar studies where landslide-tsunamis are modelled with and without frequency dispersion. The relevance of frequency dispersion on landslide-tsunami propagation is very well known and documented.

L343: The wave train is not simulated because the model is unable to model frequency dispersion. This should be mentioned/discussed.

Figure 11: The data of the numerical simulations are on the un-safe side, which maybe an issue for hazard assessment.

L381: I can not follow why the Authors state that their data are within 30\%, it looks rather like a 50\% deviation from Eq. (39)?

L384: The fact that the model cannot consider breaking also implies that it cannot consider the impact crater, etc. It would be good to mention/discuss these aspects as well.

L388-293: The argumentation in this section is misleading. The main reason for the absence of the wave train is not breaking, it is the inability of the shallow-water equation (in the form used by the Authors) to model frequency dispersion.

Printer-friendly version

Discussion paper 
L403: The Conclusions should be understandable on their own. I suggest starting with a brief motivation and method of the study before summarising the conclusions.

NHESSD

L410: I do not agree that this statement is appropriate. The wave in the numerical model behave not similar to the ones in the experiments due to the absence of frequency dispersion in the numerical model. I also do not agree with the conclusion "the choice to transfer the momentum through the simple perfectly elastic collision principle is verified to be relevant." I cannot recall where this has been shown in the article? Please clarify and/or update the Conclusions.

L416: The 211 experiments have been performed by Heller and Hager (2010), 137 ones by Fritz et al. (2002) as well as 86 ones by Zweifel (2004) (see Heller and Hager, 2010).

L417: ". . .our model is further strengthened by the fact that the results of our model also fit well with those experiments." I disagree, this statement contradicts Fig. 11. The trend of the numerical data is very different to the trend given by Eq. (39), so there is a systematic difference, and the discrepancy is up to $45 \%$.

Suggested grammatical corrections and minor points:

Title: The title includes some repetitions and should be written more concise.

L8: A landslide-generated tsunami is a water wave, it does not involve "landslide dynamics", please rephrase.

L9: Grammar issue, the plural form does not match the singular form used in the previous sentence.

L12: Please write "the shallow water...".

Printer-friendly version

L16: Please write "performs best. ..".

L19/20: Same issue as on L8.

Discussion paper 
L28: Landslide-generated tsunamis are not observed on plains, they are observed in the water body. Please rephrase.

L42: Please write "an advanced..."

L49: Please follow Harvard style by adding brackets ahead and after the year.

L54: "automatically" is not a good choice, it indicates that the applier needs to do nothing in the simulation.

L57: Consider replacing "specification" with "framework".

L62: Please write "to wave generation".

L65: "Moreover, the granular flow is gravitationally accelerated, which is a relevant aspect to test the behaviour of the numerical model." It is not fully clear what the Authors try to state with this statement, many laboratory slides are accelerated by gravity.

L68: "the comprehensive phenomenon of" is not necessary.

L71: Please replace "but" with "and".

L73: The aluminum plate and the slope are not part of the flume, they are built into the flume, please rephrase.

L75: It is common practice to write parameters in italic style in research articles, so please write $h$ in italic and also all other parameters in the text, tables and figures (e.g. $X$ in Figure 1). On the other hand, numbers (e.g. L152) must not be written in italic.

L79: Please replace "to be" with "as".

L85: Either write "an experiment" or "the experiments".

Printer-friendly version

L91: Please check the numbering style for equations and apply this style consistently throughout the manuscript. 
L97: Please check how "Section" is abbreviated (I do not think "sect." is the correct abbreviations) and apply it consistently through the manuscript.

L102: Please follow Harvard Style.

L117: "sin", "cos" and "tan" should not be written in italic, here and throughout the manuscript.

Interactive

comment

L142: Please add "the" in front of velocity.

L144: The page reference can be dropped, the relation between Chezy and Manning Coefficient is undergraduate student material.

Eq. (16): The writing style of $d 1 / 3$ seems incorrect, also the number on L154 should be written as subscript.

L151: Please write "stress at which the slide starts or stops to move".

L180/1: Please move "from our point of view" without the brackets after "coefficients".

L206: Please write "Eqs. (29) and (30)", also on L230 and L314.

Figure 2 (and the figures in general): Can the quality of the figures be improved in a professional software such as Adobe Illustrator, etc.? It looks like some of the figures are simple exported from Excel. Also please follow the consistent writing style (e.g. parameters in italic).

L220/1: There are Grammar issues here, the Authors give the impression that $A m=0$ in Miller et al. (2017) and it is also unclear what the Authors try to state with "not the best fitting curve". Why?

The Text in Fig. 3 is unreadable small.

Printer-friendly version

Table 1: Please do not use abbreviations, but rather increase the width of the table.

L264: The presentation of the paragraph can also visually be improved, e.g. by intending the first line of the paragraph. Here and throughout the article.

Discussion paper 
L276: Please write "versus" rather than "vs.".

L281: Please drop "the" in front of best.

P13 at the bottom: Please avoid such free spaces (e.g. by moving the figures), also on P16.

L292: Please drop the in front of sect. and use the correct abbreviation for Section.

Figures 7/8: The text should not be written in italic. Please also write the full term for "w/o"

L315: Please replace "through" with "to".

L316: Please drop one of the 8.

L317: Please write "a result".

L322: Please write "This section investigates the momentum transfer between the slide and the generated wave."

Fig. 9: Again, there are some issues here how the parameters/numbers are written.

Fig. 10: The same issues as elsewhere. Also replace “@” with "at”.

L349: I do not think the explanation "The coloured. .." is needed given that this is visible in the Notation. Similar statements can be dropped in figure captions elsewhere.

L357: Please write "Am is defined..."

L364: Please drop "reservoir and" as there was not variation in the reservoir.

Figure 11: Is it possible to better highlight the legend in the figure e.g. by adding a frame? The symbols in the figure may be mixed up with the real data.

Printer-friendly version

L414: Please write "the impulse product parameter particularly...", there is only one.

Discussion paper

L424: Please write "from all co-authors". 
L432: Please write "maximum measured wave amplitude"

L433: Please write "theoretical maximum wave amplitude in the near-field"

NHESSD

Notation, MTs and on the next line: Please use a more formal symbol for "->"

Notation, U: Please write "solution".

References: Please remove inconsistencies such as inconsistent use of upper and lower case letters in the article titles (e.g. L440, L534, etc.), abbreviation and no abbreviation of Journal titles, typos (e.g. L518 "genrated"), etc.

Interactive comment on Nat. Hazards Earth Syst. Sci. Discuss., https://doi.org/10.5194/nhess2019-396, 2020. 